\title{
Study on Circle Center Positioning Error by Fitting of Measurement System for Gear Chamfering
}

\author{
Zhang $\mathrm{chi}^{\mathrm{a}}$, Xu Zengpu, Liu Guohao, Wang Yongqiang and Zhou Congling \\ Department of Mechanical Engineering, Tianjin University of Science and Technology : No.1038 Dagu Nanlu,Hexi \\ District, Tianjin, China
}

\begin{abstract}
This paper briefly introduced the working principle of non-contact measurement system of gear chamfering based on computer vision technology and laser range-finding technology. Then the circle center positioning method during the process of the first-stage positioning measurement of measurement system was studied, and the influence of circle center positioning error on the precision of starting point for measurement was analyzed. The standard circle with a cross and a radius of $20 \mathrm{~mm}$ was used for the measurement experiment, as well as the precision error rule of fitting circle center positioning and influence relationship of the starting point error of measurement were studied. Thus a kind of effective method to improve the measurement precision was put forward.
\end{abstract}

\section{Introduction}

The machining precision of gear with chamfering in the automobile gearbox would directly affect the technical indexes of automobile transmission system ${ }^{[1-2]}$. At present, the non-contact measurement method based on computer vision technology and laser range-finding technology has become the main means to detect the machining precision of gear chamfering. In order to obtain the actual location of the gear, the least square method was used to fit the distribution points in the dedendum circle of gear so as to obtain the circle center coordinate of fitting circle during the actual measurement process. Whether the circle center positioning is accurate or not directly affects the subsequent positioning of starting points for measurement in the cross section of gear chamfering as well as determines the accuracy of the final measurement results. Hence, it is necessary to analyze and study the positioning precision of fitting circle center in the first-stage vision measurement system.

\section{Working Principle of Measurement System}

\subsection{Measurement object and parameters}

Figure 1 (a). showed the real picture of gear with chamfering. The main parameters to be measured were the angle $\mathrm{M}$ of the modeling section and the distance $\mathrm{N}$ from the intersecting line of modeling

a Corresponding author : zhangchi1025@163.com 
surface to the specified surface, as shown in Figure 1 (b) ${ }^{[3-4]}$.

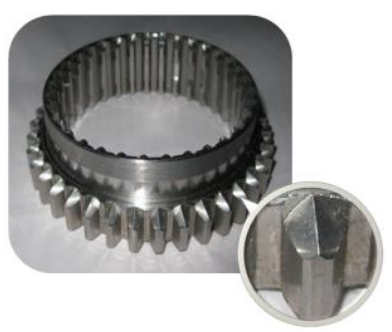

(a) Real picture of gear with chamfering.

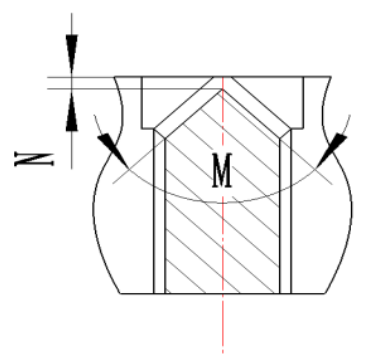

(b) Parameters to be measured.

Figure 1. Structure and measurement parameters of gear chamfering.

\subsection{Working principle of measurement system}

The non-contact measurement system of gear with chamfering was composed of XY two-dimensional precise mobile platform, camera (CCD array sensor), backward light source, laser displacement sensor, marble base, rotating stage, computer, and so on (shown in Figure 2.) This system used the two-stage collaborative measurement way. The first-stage measurement adopted both the array image sensor and calibration algorithm to complete the preliminary measurement positioning [5]. Likewise, the second-stage measurement used two-dimensional high-precision electric platform with a laser displacement sensor to finish the high-precision measurement for workpiece contour sizes according to the planning path, and then the contour and parameters of gear chamfering were worked out by program.

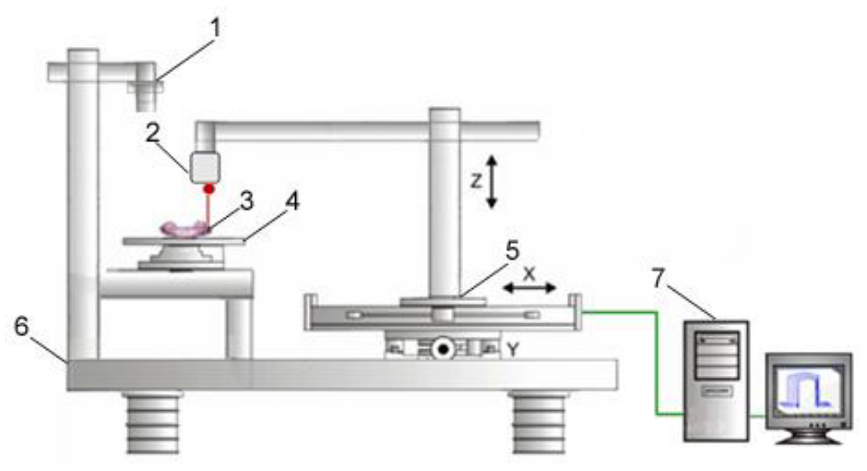

Figure 2. Structure diagram of measurement system

\subsection{Positioning method of gear circle center}

In the first-stage positioning measurement, the gray images of gear edge contour were collected by cameras, and then the images were processed by programs written by $\mathrm{VC}++6.0$ software. Furthermore, the edge detection algorithm was used to extract the coordinates set of location points for the outer circle edge of gear images under the image pixel coordinate system UV ${ }^{[6]}$. This set can be divided into three subsets according to different locations of edge points, including the points set of addendum circle, the points set of dedendum circle, and the points set of tooth profile. The points set of dedendum circle was extracted from them, and then least square circle fitting algorithm was used to obtain the coordinate of the gear circle center. The set composed of all the points on the dedendum circle was denoted by $\mathrm{D}$, and the distance from any point $\mathrm{D}_{\mathrm{i}}\left(\mathrm{u}_{\mathrm{i}}, \mathrm{v}_{\mathrm{i}}\right)$ to the circle center was $\mathrm{R}_{\mathrm{i}}$. The 
area of the circle with a radius of $\mathrm{R}_{\mathrm{i}}$ was $\mathrm{s}_{\mathrm{i}}$. In addition, the fitting circle center coordinate was $\left(\mathrm{x}_{0}, \mathrm{y}_{0}\right)$, the standard circle radius was $\mathrm{R}$, and its area was $\mathrm{s}$. Then the area error of two circles was:

$$
\begin{aligned}
& \varepsilon_{i}=s_{i}-s=\pi R_{i}^{2}-\pi R^{2} \\
& =\pi\left[\left(u_{i}-x_{0}\right)^{2}+\left(v_{i}-y_{0}\right)^{2}-R^{2}\right]
\end{aligned}
$$

The quadratic sum function of error was expressed as:

$$
F\left(x_{0}, y_{0}, R\right)=\pi^{2} \sum_{i=1}^{n}\left[\left(u_{i}-x_{0}\right)^{2}+\left(v_{i}-y_{0}\right)-R^{2}\right]^{2}
$$

According to the least squares principle, the values of $\mathrm{x}_{0}, \mathrm{y}_{0}$ and $\mathrm{R}$ were achieved as the minimum of $\mathrm{F}\left(\mathrm{x}_{0}, \mathrm{y}_{0}, \mathrm{R}\right)$ was taken. Thus the partial derivative of $\mathrm{F}\left(\mathrm{x}_{0}, \mathrm{y}_{0}, \mathrm{R}\right)$ was worked out and then was equal to zero, namely,

$$
\begin{aligned}
& \frac{\partial F}{\partial x_{0}}=\frac{\partial F}{\partial y_{0}}=\frac{\partial F}{\partial R}=0 \\
& \frac{\partial F}{\partial x_{0}}=\pi^{2} \sum_{i=1}^{n}(-4)\left[\left(u_{i}-x_{0}\right)^{2}+\left(v_{i}-y_{0}\right)^{2}-R^{2}\right]\left(u_{i}-x_{0}\right)=0 \\
& \frac{\partial F}{\partial y_{0}}=\pi^{2} \sum_{i=1}^{n}(-4)\left[\left(u_{i}-x_{0}\right)^{2}+\left(v_{i}-y_{0}\right)^{2}-R^{2}\right]\left(v_{i}-y_{0}\right)=0 \\
& \frac{\partial F}{\partial R}=\pi^{2} \sum_{i=1}^{n}(-4)\left[\left(u_{i}-x_{0}\right)^{2}+\left(v_{i}-y_{0}\right)^{2}-R^{2}\right] R=0
\end{aligned}
$$

Equation (4) was solved, then

$$
\left\{\begin{array}{l}
x_{0}=\frac{h q-t c}{2\left(p q-c^{2}\right)} \\
y_{0}=\frac{t p-h c}{2\left(p q-c^{2}\right)}
\end{array}\right.
$$

Where

$$
\begin{aligned}
& p=m \sum x_{j}^{2}-\left(\sum x_{j}\right)^{2} \\
& q=m \sum y_{j}^{2}-\left(\sum y_{j}\right)^{2} \\
& h=m \sum x_{j}^{3}+n \sum x_{j} y_{j}^{2}-\sum\left(x_{j}^{2}+y_{j}^{2}\right) \sum x_{j} \\
& t=m \sum y_{j}^{3}+m \sum x_{j}^{2} y_{j}-\sum\left(x_{j}^{2}+y_{j}^{2}\right) \sum y_{j} \\
& c=m \sum x_{j} y_{j}-\sum\left(x_{j}\right) \sum y_{j}
\end{aligned}
$$

The precise circle center coordinate $\mathrm{O}\left(\mathrm{O}_{\mathrm{u}}, \mathrm{O}_{\mathrm{v}}\right)$ of the gear could be obtained by equation (5).

\subsection{Influence of positioning error of circle center on the precision of starting point for measurement}

There existed error in the position of circle center coordinate, hence, the error would also appear in the 
starting point position of gear chamfering.

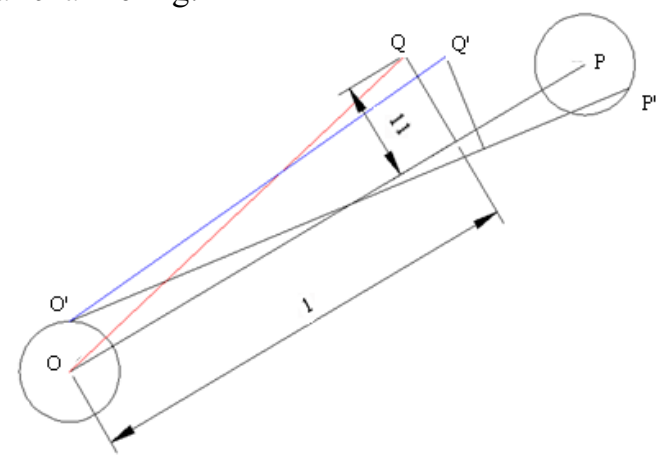

Figure 3. Analysis method of influence of circle center error on the starting points for measurement.

The coordinates of points $Q$ and $Q^{\prime}$ deduced from Figure 3 were shown in equations (6)-(9).

$$
\begin{aligned}
& Q x=\sqrt{l^{2}+l 1^{2}} \cdot \cos \left(\arctan \left(\frac{P y-O y}{P x-O x}\right)+\arctan \left(\frac{l 1}{l}\right)\right)+O x \\
& Q y=\sqrt{l^{2}+l 1^{2}} \cdot \sin \left(\arctan \left(\frac{P y-O y}{P x-O x}\right)+\arctan \left(\frac{l 1}{l}\right)\right)+O y \\
& Q^{\prime} x=\sqrt{l^{2}+l 1^{2}} \cdot \cos \left(\arctan \left(\frac{P y+\delta y-O y-\Delta y}{P x+\delta x-O x-\Delta x}\right)+\arctan \left(\frac{l 1}{l}\right)\right)+O x+\Delta x \\
& Q^{\prime} y=\sqrt{l^{2}+l 1^{2}} \cdot \sin \left(\arctan \left(\frac{P y+\delta y-O y-\Delta y}{P x+\delta x-O x-\Delta x}\right)+\arctan \left(\frac{l 1}{l}\right)\right)+O y+\Delta y
\end{aligned}
$$

Where $Q$ was the theoretical starting point for measurement. $Q^{\prime}$ was the measurement starting point deduced by fitting. $l$ was the distance from the measuring cross section to the circle center of gear. $l 1$ was half the distance from the measuring cross section to the circle center of gear. $O$ was the theoretical circle center of gear. $O^{\prime}$ was the circle center of gear by fitting. $P$ was the theoretical central point of addendum arc chord. $P^{\prime}$ was the central point of addendum arc chord obtained by fitting.

\section{Measurement Experiment}

\subsection{Experiment scheme}

In order to verify the detection precision of measurement system, the standard circle with a cross and a radius of $20 \mathrm{~mm}$ (shown in Figure 4) was used for field measurement. The experiment method was as follows. Firstly, the camera was used to take checkerboard images, and then the sizes were calibrated. After the calibration, the checkerboard was removed, and the standard circle was brought. Then the standard circle images were taken (standard circle was placed below the camera lens and kept away from the camera lens. Then the standard circle images were taken one by one.) The computer was used to process the images one by one to obtain the points on the outer contour of standard circle. Thus the circle center coordinate was obtained by fitting. Then the cross lines were manually fitted to obtain the intersection, and the intersection was taken as the theoretical circle center. Finally, the 
circle center coordinate of fitting circle was compared with the hypothetically theoretical circle center coordinate to work out the positioning precision error of the circle center coordinate of fitting circle in the measurement system, and the measurement results were shown in Table 1.

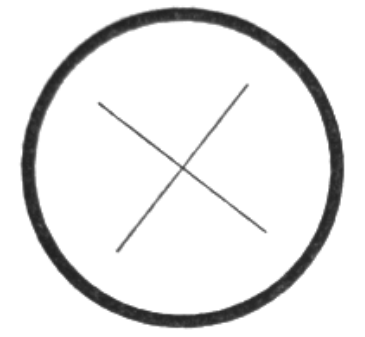

Figure 4. Standard circle with a cross.

Table1. Measurement results on error of center location in measuring

\section{Distance}

Error (mm)

\begin{tabular}{|ccccccccccc}
\hline Near & 0.03373 & 0.02236 & 0.02961 & 0.07689 & 0.081437 & 0.06933 & 0.06906 & 0.06594 & 0.06296 \\
& 0.04070 & 0.03190 & 0.01431 & 0.04472 & 0.037643 & 0.03088 & 0.05024 & 0.05930 & 0.06184 \\
& 0.09701 & 0.04687 & 0.03640 & 0.02707 & 0.027586 & 0.01216 & 0.02690 & 0.03512 & 0.06640 \\
& 0.10751 & 0.06726 & 0.00412 & 0.07905 & 0.045099 & 0.01526 & 0.02816 & 0.03417 & 0.08338 \\
& 0.08297 & 0.04812 & 0.03101 & 0.08586 & 0.087143 & 0.02213 & 0.01843 & 0.04111 & 0.09861 \\
$\underbrace{}_{\text {Distant }}$ & 0.04904 & 0.061770 & 0.03114 & 0.07975 & 0.095707 & 0.05261 & 0.02047 & 0.04494 & 0.03257
\end{tabular}

As shown in Table 1, when the standard circle moved from the near to the distant, there was no obvious change trend for the error between the fitting circle center and theoretical circle center. Hence, all the measuring data needed the statistical analysis, as shown in Figure 4.

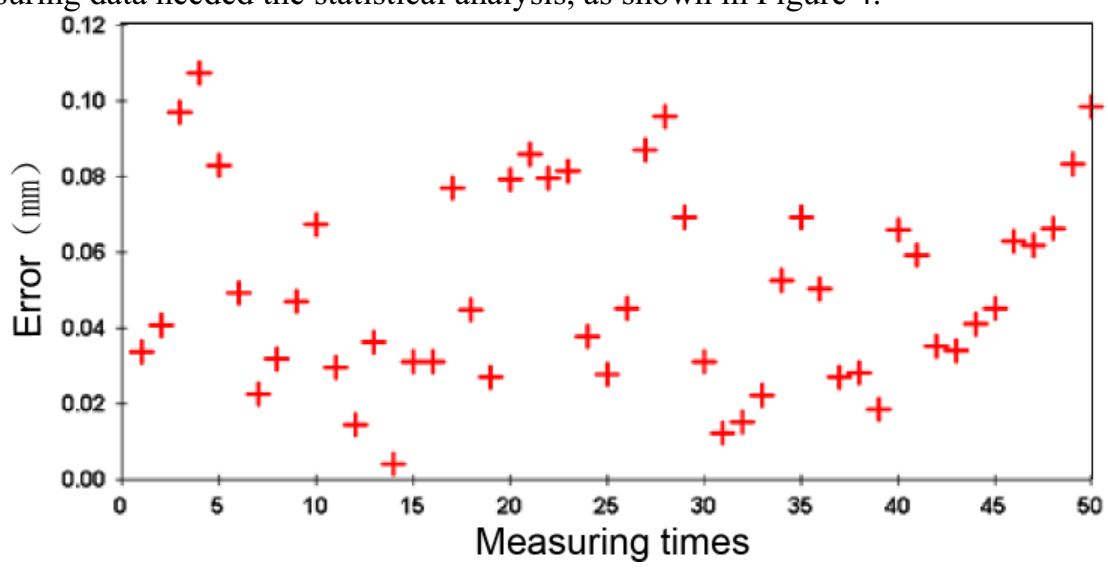

Figure 5. Measurement data error distribution of circle center positioning errors.

As shown in Figure 5, few error data distributed in two sides, the error data mostly distributed between $0.03 \mathrm{~mm}$ and $0.08 \mathrm{~mm}$, and the mean of errors was $0.0509 \mathrm{~mm}$. In order to further verify the 
influence of circle center error on the starting point position of measurement section, the software program codes written according to the experiment results were as follows.

$\mathrm{x} 1=\mathrm{r} 1 * \cos (\mathrm{sita}) ; \% \mathrm{X}$ coordinate of circle center error by fitting;

$\mathrm{y} 1=\mathrm{r} 1 * \sin (\mathrm{sita}) ; \% \mathrm{Y}$ coordinate of circle center error by fitting

$\mathrm{x} 2=0 ; \% \mathrm{X}$ coordinate of center error by fitting;

y $2=0 ; \%$ Y coordinate of center error by fitting;

$\% \quad \mathrm{x} 1=0 ; \% \mathrm{X}$ coordinate of circle center error by fitting;

$\% \quad \mathrm{y} 1=0 ; \% \mathrm{Y}$ coordinate of circle center error by fitting;

$\% \quad \mathrm{x} 2=\mathrm{r} 2 * \cos ($ beta) $; \% \mathrm{X}$ coordinate of center error by fitting;

$\% \quad \mathrm{y} 2=\mathrm{r} 2 * \sin ($ beta) $; \%$ Y coordinate of center error by fitting;

alpha1 $=\operatorname{atan}((\mathrm{p} 2-\mathrm{o} 2) /(\mathrm{p} 1-\mathrm{o} 1)) ; \%$ Angle between theoretical normal and horizontal line, and this value cannot be $\mathrm{pi} / 2(\mathrm{p} 1 !=\mathrm{o} 1)$;

alpha2=atan(1/11);\% Fixed angle;

alpha $=$ alpha $1+$ alpha $2 ; \%$ The sum of theoretical angles; line;

gammal $=\operatorname{atan}((\mathrm{p} 2+\mathrm{y} 2-\mathrm{o} 2-\mathrm{y} 1) /(\mathrm{p} 1+\mathrm{x} 2-\mathrm{o} 1-\mathrm{x} 1)) ; \%$ Angle between fitting normal and horizontal

gamma=gamma1+alpha2;\% The sum of fitting angles;

$\mathrm{x} 11=\operatorname{sqrt}(1 * 1+11 * 11) * \cos ($ alpha $)+01 ; \% \quad \mathrm{X}$ coordinate of theoretical starting points for measurement;

$\mathrm{y} 11=\operatorname{sqrt}(1 * 1+11 * 11) * \sin ($ alpha $)+02 ; \% \quad \mathrm{Y}$ coordinate of theoretical starting points for measurement; fitting;

$\mathrm{x} 22=\operatorname{sqrt}(1 * 1+11 * 11) * \cos ($ gamma $)+o 1+x 1 ; \% \mathrm{X}$ coordinate of starting points for measurement by

$\mathrm{y} 22=\operatorname{sqrt}(1 * 1+11 * 11) * \sin ($ gamma $)+\mathrm{o} 2+\mathrm{y} 1 ; \% \mathrm{Y}$ coordinate of starting points for measurement by fitting;

$\mathrm{d}=\operatorname{sqrt}\left(\left((\mathrm{x} 11-\mathrm{x} 22)^{\wedge} 2\right)+\left((\mathrm{y} 11-\mathrm{y} 22)^{\wedge} 2\right)\right) ; \%$ Distance between theoretical points and fitting points;

The programs were run, and then the analysis results were obtained, as shown in Table 2.

Table 2. Effect of central fitting error on the starting points for measurement.

\begin{tabular}{cccccccc}
\hline Name & \multicolumn{7}{c}{ Measurement data } \\
\hline Circle center error $(\mathrm{mm})$ & 0.01 & 0.02 & 0.03 & 0.04 & 0.05 & 0.06 \\
$\begin{array}{l}\text { Error of starting points for } \\
\text { measurement }(\mathrm{mm})\end{array}$ & 0.01013 & 0.02025 & 0.03038 & 0.04050 & 0.05063 & 0.06076 \\
\hline
\end{tabular}

It can be seen from the measuring data that the error of circle center fitting was proportional to the error of starting points for measurement. Namely, the error of starting points for measurement was about 1.013 times as large as the error of circle center. The camera pixel was equivalent to the actual size of $0.178 \mathrm{~mm}$, and the measuring error was within a pixel. Hence, in order to improve the circle center precision of fitting circle, the camera and lens with high resolution should be chosen so as to reduce pixel minimal size and decrease circle center error of fitting circle, and thus the positioning precision of starting points for measurement was improved.

\section{Conclusions}

This paper studied the circle center positioning precision of fitting circle in the non-contact measurement system of gear with chamfering and determined that the precision of fitting circle center was $0.0509 \mathrm{~mm}$ in the measurement system by the experiment. Then the influence of circle center positioning error on the position errors of starting points for measurement on the measuring cross section was analyzed. Finally, it is concluded that the fitting precision of circle center was improved by improving the resolution of camera and decreasing the distinguishable minimal size precision. This conclusion can provide the theoretical basis for perfecting the working performance and measuring precision of measurement system. 


\section{References}

1. Xu Zengpu, Zhang Chi, Cai Chunming, and et al. Study on cooperative high precision measurement method of gear chamfering profile. Manufacturing Automation [J] ( 2015)

2. Geometrical modeling and adjustment simulation of the chamfering cutter of gear curved face gear, written by Zhanwen Zhu, Renlong Liu, Wei Guo, Tianjin University Paper (2006)

3. XU Z P, ZH SH. Gear Chamfering Profile Measurement System Based On Laserb And Machine Vision [C]. AMR, [EI] (2011)

4. LI Z Q, DONG X L, LI Q L, GUO C L. Research of small modulus gear vision measurement system $[\mathrm{J}]$.Coastal enterprise and science \& technology (2010)

5. XU Z P, YU D M. Auto-calibration method of light and image plates [J]. Journal of Tianjin institute of light industry (1993)

6. C Zhou, Z Xu, C Cai, Y Wang, A high precision collaborative vision measurement of great chamfering profile[C],International Conference on Advances in Mechanical Engineering \& Industrial Informatics (2015) 